\title{
RIGOR MORTIS E ASPECTOS REPRODUTIVOS DE ACARIS BODÓS CAPTURADOS NO RIO TAPAJÓS, PARÁ, BRASIL
}

\author{
Mateus Levi Avelino Moura'; Felipe Takis Cunha1; Hugo José Amorim de Macedo1; Jamille \\ Débora De Oliveira Batista²; Samuel Maia Reis ${ }^{1}$; Sullyvan Silva Oliveira'; Fabrizia Sayuri Otani ${ }^{3}$. \\ 1Universidade Federal do Oeste do Pará, Santarém, Pará, Brasil, mateuslevi1995@gmail.com \\ ¿Universidade Federal da Grande Dourados, Dourados, MS, Brasil, jamilledeboraob@gmail.com \\ 3Universidade Federal do Oeste do Pará, Santarém, Pará, Brasil, fabrizia.otani@ufopa.edu.br
}

RESUMO: Este trabalho teve como objetivo analisar alguns aspectos da biologia reprodutiva de acaris-bodós, Ptrygoplichthys pardalis, avaliando o fator de condição de Fulton (k), índice gonadossomático (IGS), proporção sexual e estágio de maturação sexual, e também objetivou-se avaliar a resolução do rigor mortis, nos peixes sacrificados em condições estressantes. Vinte peixes foram capturados no rio Tapajós, sendo 10 sacrificados imediatamente e 10 sacrificados sob condições de estresse pelo transporte, para avaliação do rigor mortis. Também se mensurou peso e comprimento total para determinação do fator de condição de Fulton ( $k$ ), assim como foram coletadas as gônadas, sexadas, e pesadas, para determinação do índice gonadossomático (IGS) e proporção sexual, e classificadas quanto ao estágio de maturação sexual. Os peixes sacrificados após estresse atingiram o rigor mortis em menos tempo, em relação aos peixes sacrificados imediatamente. Os acaris-bodós estavam em bom estado nutricional, apresentando valores de $k$ de 1,02 $\pm 0,08$, proporção sexual de fêmeas:machos de 9:1, índice gonadossomático (IGS) de 0,244 $\pm 0,068 \%$, e apresentaram estágio de maturação sexual como fêmeas desovadas ou esvaziadas, no período chuvoso.

PALAVRAS-CHAVES: Organismo aquático, Pterygoplichthys pardalis, Reprodução.

\section{RIGOR MORTIS AND REPRODUCTIVE ASPECTS OF ACARIS BODÓS CAPTURED IN THE TAPAJÓS RIVER, PARÁ, BRAZIL}

ABSTRACT: This work aimed to analyze some aspects of the reproductive biology of acaris-bodós, Ptrygoplichthys pardalis, evaluating the Fulton condition factor (k), gonadosomatic index (IGS), sexual ratio and stage of sexual maturation, and also aimed to evaluate the resolution of rigor mortis, in fish sacrificed under stressful conditions. Twenty fish were captured in the Tapajós River, 10 of which were immediately killed and 10 were sacrificed under transport stress conditions for rigor mortis evaluation. We also measured weight and total length for determination of the Fulton condition factor ( $k$ ), as well as the gonads, sexed, and heavy, for determination of the gonadosomatic index (IGS) and sex ratio, and classified for the maturation stage 
sexual. Fish slaughtered after stress reached rigor mortis in less time, in relation to fish slaughtered immediately. The fish were in good nutritional status, presenting $k$ values of 1,02 $\pm 0,08$, sex ratio of females: males of 9: 1 , gonadosomatic index (IGS) of 0,244 $\pm 0,068 \%$, and presented a maturation stage as females spawned or emptied in the rainy season.

KEYWORDS: Aquatic organism, Pterygoplichthys pardalis, Reproduction.

\section{RIGOR MORTIS Y ASPECTOS REPRODUCTIVOS DE ACARIS BODÓS CAPTURADOS EN EL RÍO TAPAJÓS, PARÁ, BRASIL}

RESUMEN: Este trabajo tuvo como objetivo analizar algunos aspectos de la biología reproductiva de acaris-bodós, Ptrygoplichthys pardalis, evaluando el factor de condición de Fulton (k), índice gonadosomático (IGS), proporción sexual y etapa de maduración sexual, y también se objetivó evaluar la resolución del rigor mortis, en los peces sacrificados en condiciones estresantes. Veinte peces fueron capturados para la recolección de los datos. En el rigor mortis, acaris-bodós sacrificados bajo estrés alcanzaron el rigor mortis antes de peces no sometidos al estrés. Para el factor de condición de Fulton ( $K$ ) los peces estaban en buen estado nutricional, presentando valor de 1,02 \pm 0,08 y para el índice gonadosomático (IGS) hembras de P. pardalis de $0,244 \pm 0,068 \%$ y presentaron etapa de maduración sexual como hembras desovadas o vaciadas, en el período lluvioso, con proporción sexual 9: 1.

PALABRAS-CLAVE: Organismo acuático, Pterygoplichthys pardalis, Reproducción.

Os corpos hídricos amazônicos possuem vasta diversidade de espécies de peixes, por sua riqueza em alimento e grande extensão territorial, que apresenta 3.889.489,6 $\mathrm{Km}^{2}$ de extensão, sendo considerada a maior superfície de água do mundo. Os peixes de água doce estão entre os mais importantes organismos que compõem o nécton de ambientes lóticos e lênticos, e têm sido cada vez mais estudados devido importância na cadeia trófica e dinâmica reprodutiva, além de ser importante fonte alimentar e de nutrientes como proteínas, minerais e vitaminas (BRAGA et al, 2006). O estado do Pará é o maior produtor de pescado do Brasil, tendo o segundo maior consumo per capita do 
país de pescado, com cerca de 24 kg/ano (BRASIL, 2014).

Dentre estas espécies, destaca-se o Pterygoplichthys pardalis, popularmente conhecido como acari-bodó ou cascudo no estado do Pará que é importante para fabricação de farinha de peixe para consumo humano. Pertencente à ordem dos Siluriformes, família Loricariidae (REIS et al., 2003). Os membros dessa família apresentam corpo roliço ou achatado em seção transversal e coberto por placas ósseas, formando três a cinco séries sobre o tronco, sendo cada uma munida de pequenas estruturas ósseas, bastante ásperas ao tato ou mesmo perfurantes, denominados odontódios (SANTOS et al., 2006). Apresentam região abdominal achatada ou plana e uma boca ventral que justifica seu hábito alimentar iliófago. Não apresentam linha lateral, distinguindo-se da maioria das espécies de bodós pelo grande número de raios da nadadeira dorsal, que vão de 12 a 14, e podem alcançar $50 \mathrm{~cm}$ de comprimento (SANTOS et al., 2006; PORTO et al., 2011).
Dentre os produtos de origem animal, o pescado é bastante suscetível aos processos de deterioração, sob a influência de fatores fisiológicos, químicos e microbiológicos. Sua decomposição está associada a características intrínsecas, como rápido desenvolvimento do rigor-mortis, constituição frouxa do tecido conectivo e por ser um produto rico em proteína e fosfolipídeos (BRESSAN, 2001). Uma das etapas importantes para se manter a qualidade do pescado é o abate (RIBAS et al., 2007).

Para transformação do músculo em carne, são necessárias transformações bioquímicas, iniciadas pelo processo denominado rigor mortis. Esse fenômeno se inicia após a morte do animal, sendo a primeira transformação que ocorre no peixe, seguido pela ação autolítica das enzimas musculares e a ação dos micro-organismos, culminando com a total deterioração da qualidade do pescado. Assim, o retardo do início do rigor mortis é benéfico para manutenção do frescor do pescado (FONTENELE, 2013). A condição 
energética corporal dos peixes pode influenciar no desenvolvimento gonadal (LE CREN, 1951). Para determinação de condição energética corporal, é possível utilizar medidas morfológicas, como medidas estruturais e de peso, e suas combinações, na forma de índices. 0 fator de condição de Fulton ( $k$ ) é o índice mais utilizado em peixes, em que é relação entre peso e comprimento, fornecendo estimativa indireta do armazenamento energético dos peixes (CAMARA et al., 2011; NASH et al., 2006).

Quando se trata do processo reprodutivo dos peixes, ele pode ser influenciado por fatores exógenos e endógenos, como temperatura, níveis dos corpos hídricos, alterações físicoquímicas da água, entre outros (DUTRA et al., 2010). E em relação à biologia reprodutiva, os acaris bodós têm fecundação e desenvolvimento externo e podem apresentar desova parcelada, chegando a desovar até três vezes por ano. O período específico de desova dessa espécie acontece entre o final da seca e o início da enchente (SANTOS et al., 2006; VAZZOLER, 1996). Para determinação do período reprodutivo de uma espécie, métodos qualitativos podem ser empregados, como o índice gonadossomático (IGS), que expressa em porcentagem o peso das gônadas em relação ao peso total do corpo dos indivíduos (VAZZOLER, 1996). O índice gonadossomático (IGS) é um dado auxiliar utilizado na determinação dos estádios do ciclo reprodutivo de peixes, devido a maturação das células reprodutivas ocorrer concomitantemente com o aumento do peso das gônadas (PEREIRA et al., 2004).

Assim o objetivo do presente trabalho analisar alguns aspectos da biologia reprodutiva de acaris-bodós, Pterygoplichthys pardalis (CASTELNAU, 1855), coletados no rio Tapajós, estado do Pará, avaliando o fator de condição de Fulton (k), índice gonadossomático (IGS), proporção sexual e estágio de maturação sexual, e também objetivouse avaliar a resolução do rigor mortis, nos peixes sacrificados em condições estressantes.

Foram coletados 20 peixes da espécie Pterygoplichtys pardalis (CASTELNAU, 
1855), no rio Tapajós, região oeste do Pará, na cidade Santarém Pará, localizada a 51m de altitude, $02^{\circ}$ 26'35" latitude e $54^{\circ} 42^{\prime} 30^{\prime \prime} \mathrm{W}$ de longitude, em junho de 2016. Os peixes foram anestesiados por insensibilização em água e gelo, na proporção de 1:1, e sacrificados por meio de perfuração dos arcos branquiais, no laboratório de Tecnologia de Produtos de Origem Animal (LTPOA), do Instituto de Biodiversidade e Florestas, Universidade Federal do Oeste do Pará - UFOPA.

Os peixes foram pesados, em balança semi-analítica, de precisão 0,1g, mensurou-se comprimento total, com auxílio de paquímetro digital Marberg ${ }^{\circledR}$. Para a determinação do índice de rigor mortis, cada peixe de cada grupo foi medido segundo Bito et al. (1983), inicialmente logo após o abate (T1), após 30 minutos (T2), e a cada hora até que atingisse $100 \%(T 1, T 2, T 3, T 4, T 5, T 6, T 7)$, e o índice de rigor mortis foi determinado pela equação:

$$
\text { Índice de rigor (\%) }=[(\mathrm{D} 0-\mathrm{Dt}) / \mathrm{DO}] \times 100
$$

Onde D0 e Dt representam a distância da base da nadadeira caudal à linha horizontal da mesa no início do pré-rigor e durante a estocagem.
As médias do índice de rigor mortis de G1 e G2 foram submetidas ao teste estatístico, no programa estatístico Statistical Analysis System (SAS versão 9.1). As médias entre G1 e G2 foram comparadas pelo teste t-Student, ao nível de 5\% de significância. Para os valores do fator de condição de Fulton (k) os resultados foram tabulados em planilhas do programa Microsoft Excel ${ }^{\circledR}$, do pacote de programas Microsoft Office ${ }^{\circledR}$, para cálculo de média e desvio padrão. Com os valores de média e desvio padrão também se calculou o Fator de Condição de Fulton (k), segundo fórmula:

$(k)=102 \times$ [peso total $(\mathrm{kg}) \times$ comprimento total-3(cm)]

Com auxílio de paquímetro manual, para biometria, e eviscerados por seção transversal, com auxílio de bisturi e tesoura cirúrgica, para coleta das gônadas, que foram pesadas em balança analítica, de precisão 0,01 mg e medidas, com auxílio de paquímetro digital Marberg ${ }^{\circledR}$. Para identificação do sexo e classificação do estágio de 
maturação gonadal, por identificação macroscópica, utilizou-se classificação segundo Vazzoler (1996). Para o cálculo do índice gonadossomático (IGS), foi utilizada a fórmula IGS = (PG/PT) x100, sendo PG a massa da gônada, e PG a massa do peixe.

O rigor mortis foi atingido primeiro pelo grupo que foi submetido ao manejo estressante antes do sacríficio (G2), no tempo 5 (T5), correspondendo ao período de três horas e trinta minutos após o abate (Figura 1). A partir de T2, todos os tempos diferiram estatisticamente $(p<0,05)$. O grupo sem estresse (G1) atingiu o rigor mortis em cinco horas e trinta minutos.

Esta espécie é comercializada viva, justificada pelos pescadores como uma espécie que deteriora rapidamente. Mesmo sem o manejo de estresse, ambos os peixes atingiram o rigor mortis em menos tempo, comparado com Fontenelle et al. (2013), que obtiveram maiores valores de índice de rigor mortis após 77 horas do abate, em tilápias do Nilo.

Figura 1. Índice de rigor mortis (em porcentagem) de acaris-bodós Pterygoplichthys pardalis, abatidos sem estresse (G1) e submetidos ao estresse antes do abate (G2), avaliados logo após abate (T1), após 30 minutos (T2), e após cada hora $(T 3, T 4, T 5, T 6, T 7)$.

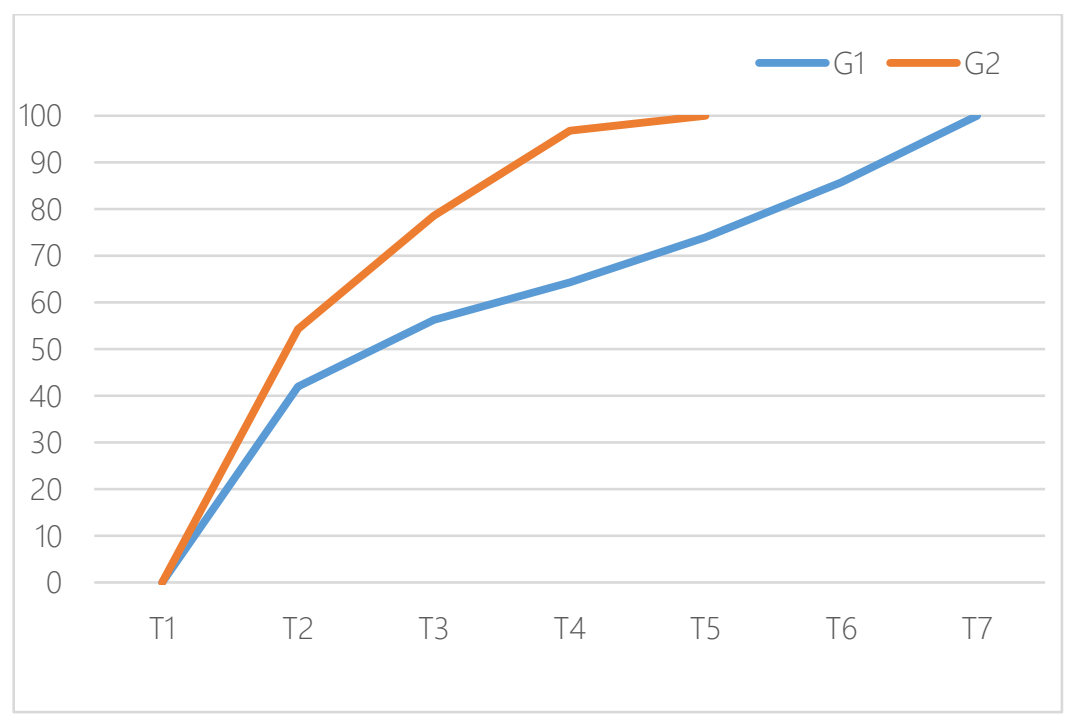


Os peixes capturados apresentaram peso médio de 289,0 \pm 36,0 g, comprimento total de $30,5 \pm 1,4 \mathrm{~cm}$, e valor do fator de condição de Fulton (k) de 1,02 \pm 0,08. $\bigcirc k$ utiliza medidas de variação de peso e comprimento, como indicadores de bem-estar geral, gordura e desenvolvimento gonadal (LE CREN, 1951). Valores de $k$ próximos de 1 (um) indicam melhor qualidade nutricional (SANT'ANA et al., 2010). Comparando com resultados encontrados para cachara (Pseudoplatystoma reticulatum) (OTANI, 2012), verificou-se que os valores de $k$ são aproximados, sendo que dos cacharas foi de 1,10 \pm 0,08, que foram capturados em Léndario, rio Paraguai. Os acaris-bodós alimentam-se de matéria orgânica particulada, como protozoários, fungos e bactérias, enquanto que os cacharas são ictiófagos, com uma variedade intensa de espécies predadas (CREPALDI et al., 2006; SANTOS et al., 2006), assim as diferenças morfológicas podem ser pelo tamanho e sua alimentação (ALASALVAR et al., 2002). Com os resultados, sugere-se que os peixes estão em bom estado nutricional, devido valor de k elevado.

Para a análise do índice gonadossomático (IGS), 18 fêmeas analisadas, o peso médio foi de $293,33 \pm 33,25 \mathrm{~g}$, comprimento total de 30,69 $\pm 1,25 \mathrm{~cm}$. As gônadas das fêmeas são pares, alongadas e foliáceas, e os ovários foram classificados em estágio de maturação gonadal em esvaziadas ou desovadas. Os ovários se apresentaram flácidos, vascularizados e com presença de regiões hemorrágicas. Foi possível visualizar ovócitos a olho nu, predominando os de estoque de reserva, e alguns desenvolvidos que não foram liberados. Trabalhos indicam que o período específico de desova dessa espécie acontece entre o final da seca e o início do período chuvoso (SANTOS et al., 2006; VAZZOLER, 1996). Constroem seus ninhos que podem ser buracos ou uma depressão localizada no fundo de lagos, próximos as margens dos rios ou em barrancos, sua fecundidade está em torno de 1000 a 5000 ovócitos. Seu período de reprodução é considerado 
longo, pois apresentam desova parcelada, sendo de 2 a 3 anuais e cuidam da ninhada até a eclosão dos ovos (SANTOS, 2006).

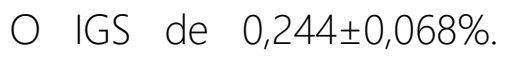

índice gonadossomático faz relação entre porcentagem do peso das gônadas para o peso total do corpo dos peixes (VAZZOLER, 1996). A análise de índice gonadossomático em peixes, auxilia a interpretar a influência de fatores ambientais e das características morfológicas em relação ao ciclo reprodutivo dos peixes, subsidiando informações para conservação e preservação de estoques pesqueiros, assim como, servindo de base para futuros trabalhos visando preservar estas espécies como também a variabilidade genética (DUTRA et al., 2010). O ápice de uma atividade reprodutiva pode ser indicado pela relação gonadossomática, enquanto que a análise dos estádios gonadais indica o período reprodutivo (JUNE, 1953). Há poucos estudos sobre a reprodução de acaris-bodós, assim os valores obtidos no presente trabalho podem nortear mais estudos comparando outros períodos sazonais e estágios de maturação gonadal de fêmeas de Pterygoplichthys pardalis.

As gônadas das fêmeas são pares, alongadas e foliáceas, e os ovários foram classificados em estádio de maturação gonadal em esvaziadas, em que os ovários se apresentaram flácidos, vascularizados e com presença de regiões hemorrágicas. Foi possível visualizar ovócitos a olho nu, predominando os de estoque de reserva, e alguns desenvolvidos que não foram liberados. Os testículos são pares, alongados, foliáceos com bordas irregulares, e foram classificados como esgotados (VAZZOLER, 1996). As gônadas apresentaram peso médio de 0,70 \pm 0,24 g, e comprimento médio de 1,99 $\pm 0,38 \mathrm{~cm}$. Dos 20 peixes analisados, 18 acaris bodós foram identificados como fêmeas, e 2 machos, sendo a proporção sexual 9:1.

Os resultados obtidos no presente trabalho da morfologia macroscópica foram descritos como a maioria dos 
trabalhos com teleósteos. $\bigcirc$ pico de uma atividade reprodutiva pode ser indicado pela relação gonadossomática, enquanto que a análise dos estádios gonadais indica o período reprodutivo (JUNE, 1953).

Assim, os resultados do presente trabalho indicaram que os peixes não estavam no pico da atividade reprodutiva, pela porcentagem baixa do IGS, e mais estudos são necessários ao longo do ciclo hidrológico, para ampliar o conhecimento da biologia reprodutiva da espécie.

Acaris-bodós Pterygoplichthys pardalis não estão no auge da atividade reprodutiva no final do período chuvoso, e apresentam bom estado nutricional. Em relação ao rigor mortis, acaris-bodós estressados apresentam menor tempo de resolução do rigor que peixes sacrificados imediatamente após coleta. Há poucos estudos sobre a biologia e reprodução de acaris-bodós, assim os valores obtidos no presente trabalho podem nortear mais estudos comparando outros períodos sazonais e estágios de maturação gonadal de fêmeas de Pterygoplichthys pardalis.

\section{REFERÊNCIAS}

ALASALVAR, C.; TAYLOR, K. D. A.; ZUBCOV, E.; SHAHIDI, F.; ALEXIS, M. Differentiation of cultured and wild sea bass (Dicentrarchus labrax): total lipid content, fatty acid and trace mineral composition. Food Chemistry, Amsterdam, n. 79, p. 145-150, 2002.

BRAGA, B.; TUNDISI, J. G.; REBOUÇAS, A. C. Águas doces no Brasil: Capital ecológica. Uso e conservação. $4^{a}$ edição. São Paulo - Escritoras. 2006. $732 p$.

BRASIL. Ministério da Pesca e Aquicultura. 2014. Plano de Desenvolvimento Sustentável. Cartilha Amazônia Sustentável. Disponível em: <http://www.mpa.gov.br/files/Docs/Pla nos_e_Politicas/Amazonia\%20Sustenta vel\%20Final.pdf> Acesso em: 02 abr. 2015.

BRESSAN, M.C. Tecnologia de póscolheita em peixes. 2001. $106 f$. Monografia (Especialização em Piscicultura) - Curso de pós-graduação "lato sensu" à distância, Universidade de Lavras, Lavras, MG.

CAMARA, E. M.; CARAMASCHI, E. P.; PETRY, A. C. Fator de condição: bases conceituais, aplicações e perspectivas de uso em pesquisas ecológicas com peixes. O ecologia Australis, Rio de Janeiro, v. 15, n. 2, p. 249-274, 2011. 
CREPALDI, D. V., FARIA, P. M. C., TEIXEIRA, E. D. A., RIBEIRO, L. P., COSTA, A. A. P., MELO, D. D., LOPES, V. E. O surubim na aquaculture do Brasil. Revista Brasileira de Reprodução Animal, Belo Horizonte, v. 30, n. 3/4, p.150-158, jul./dez. 2006.

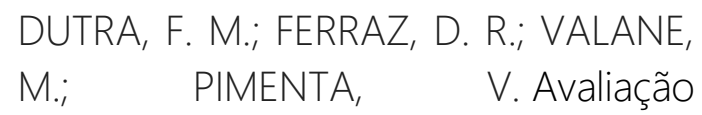
gonadossomática (IGS), em espécies de cascudos viola (Harttia loricariformis) e cascudo pintado (Hypostomus sp) em ambiente lótico e lêntico no rio Itapemirim, Alegre-ES. In: SIMPÓSIO NACIONAL DE ENGENHARIA DE PESCA, 2., SEMANA ACADÊMICA DE ENGENHARIA DE PESCA, 7., 2010. Anais... Cascavel: Unioeste, 2010. 7p.

FONTENELLE G.; KNOFF M.; FELIZARDO, N. N.; LOPES, L. M. S., São Clemente SC. Nematodes of zoonotic importance in Cynoscion guatucupa (Pisces) in the State of Rio de Janeiro. Revista Brasileira de Parasitologia Veterinária, v. 22, n. 2, p. 281-284 2013.

JUNE, F. C. Spawing of yellowfin tuna in Hawaiian Waters. Fish and Wildlife Service. Fish Bulletin. Washington, v. 54, p. 47-64, 1953.

LE CREN, E. D. The lenghtweightrelationshipandseasonalcycle in gonadweightandcondition in theperch (Perca fluviatilis). Journal of Animal Ecology, Oxford, v. 20, n. 2, p. 201-219, 1951.

NASH, R. D. M.; VALENCIA, A. H.; GEFFEN, A. J. The origin of Fulton's condition factor - setting therecordsstraight. Fisheries, Bethesda, v. 31, n. 5, p. 236-238, 2006.

OTANI, F. S. Isótopos estáveis e composição química de cacharas, Jaboticabal, 2012, 62 f., 2012. Tese (Doutorado em aquicultura) Universidade Estadual Paulista Júlio de Mesquita Filho, Centro de Aquicultura da Unesp, Jaboticabal, 2012.

PEREIRA FILHO, H. P.; DE ANDRADE, D. R.; TONINI, W. C. T.; VIDAL, M. V. V, Jr. Biologia reprodutiva de fêmeas de lambari-prata, Astyanax scabripinnis Jenyns, $1842 \quad$ (Characidae; Tetragonopterinae; Teleostei) em condições de cativeiro. Ciência Animal Brasileira, v. 12, n. 4, p. 625-634, dez. 2011.

PEREIRA, B. L.; CINTRA, B. FONSECA, V. E. LUNA, H. S.; SIMPAN, H. S. Índice gonadossomático como indicador do período Reprodutivo de Prochilodus lineatus (pisces, characidae) nos Rios Aquidauana e Miranda, MS. In: SIMPÓSIO SOBRE RECURSOS NATURAIS E SÓCIO-ECONÔMICOS DO PANTANAL, 4., 2004. Anais... Corumbá/MS. 2004.

PORTO, D. B.; VITAL, J. F.; SANTOS, A. K. S.; MORAIS, A. M.; VARELLA, A. M. B.; OLIVEIRA MALTA, J. C. Metazoários parasitos de Pterygoplichthys pardalis (Castelnau, 1855) (Siluriformes: Loricariidae) da Amazônia central, Brasil. Revista Brasileira de Zoociências, v. 14, n. 1, 2, 3, 2011.

REIS, R. E.; KULLANDER, S. O.; FERRARIS, C. J. Check list of the fresh water fishes 
of the South America. Porto Alegre: EDIPUCRS, 2003. 742p.

RIBAS, L.; FLOS, R.; REIG, L.; MACKENZIE, S.; BARTON, B.A.; TORT, L. Comparison of methods for anaesthetizing Senegal sole (Solea senegalensis) before slaughter: stress responses and final product quality. Aquaculture, v. 269, p. 250-258, 2007.

SANT'ANA, L. S.; DUCATTI, C.; RAMIRES, D. G. Seasonal variations in chemical composition and stable isotopes of farmed and wild Brazilian freshwater fish. Food Chemistry, Amsterdam, n. 122, p. 74-77, 2010.

SANTOS, G. M. D.; EFREM, J.; FERREIRA, G.; ZUANON, J. A. S. Peixes comerciais de Manaus. Manaus, AM. Ibama/AM, pró várzea, 2006. 146p.

VAZZOLER, A. E. Biologia da reprodução de peixes teleósteo: teoria e prática. Maringá: EDUEM, 1996, 169p. 